\title{
Stabilisation of amorphous calcium phosphate in
}

\section{polyethylene glycol hydrogels}

Manuel Schweikle ${ }^{a, *}$, Sindre H. Bjфrn $\phi y^{b}$, Antonius T. J. van Helvoort ${ }^{b}$, Håvard J. Haugen ${ }^{a}$, Pawel Sikorski ${ }^{b}$, Hanna Tiainen ${ }^{a}$

${ }^{\text {a }}$ Department of Biomaterials, Institute of Clinical Dentistry, University of Oslo,

Geitmyrsveien 69-71, 0455 Oslo, Norway.

${ }^{\mathrm{b}}$ Department of Physics, Norwegian University of Science and Technology (NTNU),

Høgskoleringen 5, 7491 Trondheim, Norway.

* Corresponding author: Manuel Schweikle; manuel.schweikle@odont.uio.no; +4746220722;

Department of Biomaterials, Institute of Clinical Dentistry, University of Oslo, Geitmyrsveien 69-71, 0455 Oslo, Norway 


\section{Abstract}

Acellular polymer-calcium phosphate composites are promising bone graft materials. Hydrogels are suitable for providing a temporary matrix, while calcium phosphate minerals serve as ion depots for calcium and phosphate required for de novo bone formation. Crystalline calcium phosphates are stable under biological conditions and are commonly used in such scaffolds. However, the low solubility of these phases reduces the availability of free ions and potentially obstructs the remodelling necessary for the formation of mineralised tissue. Here, we investigate two different strategies to stabilise amorphous calcium phosphates in a synthetic polyethylene glycol-based hydrogel matrix.

In vitro experiments mimicking an injectable application showed that amorphous calcium phosphate $(\mathrm{ACP})$ of variable stability was formed in the hydrogel matrices. In additive-free composites, ACP transformed into brushite within minutes. Citrate or zinc additives were found to stabilise the formed ACP phase to different degrees. In the presence of citrate, ACP was stable for at least $2 \mathrm{~h}$ before it transformed into hydroxyapatite within 3-20 days. Partial calcium substitution with zinc $(\mathrm{Zn} / \mathrm{Ca}=10 \%)$ produced zinc-doped ACP of high stability that did not show signs of crystallisation for at least 20 days.

The presented methods and findings open new possibilities for the design of novel injectable synthetic bone graft materials. The possibility to produce ACP with tailorable stability promises great potential for creating temporary scaffolds with good osteogenic properties.

\section{Keywords}

Mineralisation, composite, ACP, bone graft substitute, characterisation 


\section{Introduction}

Synthetic bone scaffolds are needed to substitute biologically sourced materials, allowing for safer, cheaper, and faster bone grafting procedures [1-4]. Calcium phosphate (CaP)-hydrogel composites are a rational choice for scaffolds intended for early tissue regeneration [5]. The hydrogel mimics a blood clot in serving as a temporary matrix, providing mechanical stability and traction for migrating cells from adjacent tissues that gradually degrade the scaffold, replacing it with new bone [6]. CaPs supply the building material for the inorganic component of the forming bone. Typically, CaP-hydrogel composites employ crystalline $\mathrm{CaP}$ phases such as nanosized hydroxyapatite (HA) [7, 8]. However, these phases are stable under physiological conditions, lack the osteoinductivity of auto- or allogeneic bone grafts [2], and are poorly remodelled into the low-crystalline apatite, which constitutes biological bone mineral [9]. Amorphous CaPs (ACP) have the potential to overcome this limitation. However, to design synthetic hydrogel-ACP scaffolds, a better understanding of the $\mathrm{CaP}$ formation and transformation in synthetic polymer matrices is required.

$\mathrm{ACP}$ is a central precursor in biomineralisation in vivo $[10,11]$, where its formation, stabilisation, and transport are highly controlled [12]. Intracellular ACP has been identified as a key factor in osteogenesis $[13,14]$, while it serves extracellularly as a precursor phase for apatite in the mineralisation of collagen fibrils $[15,16]$. The comparatively low stability of ACP means that it features exceptionally high bioactivity and biodegradation [17], making it one of the best third-generation biomaterials [18]. The amorphous particles serve as seeds for the low crystalline apatite of biogenic bone and are easily resorbed, liberating calcium and phosphate ions as they are required for de novo bone formation.

The metastable character of ACPs under biological conditions means that they need to be stabilised to be used in a composite scaffold. While ACP readily forms during precipitation [19, 
20], it freely transforms into thermodynamically favourable crystalline phases via surface nucleation followed by solution-mediated transformation [21, 22]. Two principal mechanisms can be employed to retard this crystallisation: surface adsorption of foreign molecules and ionic substitution [23]. Common organic molecules stabilising ACP by surface adsorption are ATP, poly-L-lysine, or citrate, while ions like $\mathrm{Mg}^{2+}, \mathrm{Zn}^{2+}, \mathrm{P}_{2} \mathrm{O}_{7}^{4-}, \mathrm{CO}_{3}{ }^{-}$, and $\mathrm{F}^{-}$can substitute calcium or phosphate ions in CaPs [20, 24-26].

For bone scaffolds, citrate and zinc represent two particularly suitable strategies for stabilising ACP in inert synthetic hydrogel matrices. Citrate is present in bone in concentrations of $1-$ 2 wt. $\%[9,27]$, where it plays a key role in regulating bone nanostructure and crystallinity [28]. It adsorbs to the surface of ACP particles, where it retards crystallisation by interfering with surface nucleation [29]. Low concentrations of zinc are present in bone tissues $(\mathrm{Zn} / \mathrm{Ca} \sim 1.5-$ $2.2 \mu \mathrm{g} / \mathrm{mg}$ [30]), contributing to bone homeostasis [26, 31]. Of all common ionic substitutes in biological CaPs, $\mathrm{Zn}^{2+}$ has the highest affinity to the phosphate ion [32]. The smaller zinc ion readily substitutes calcium in amorphous and crystalline CaPs [33], inhibiting crystallisation by distorting the atomic order and decreasing solubility [34, 35]. While cytotoxic in high concentrations [36], small amounts of zinc are known to have an osteogenic effect, promoting bone remodelling [31, 37-39].

The aim of this study is twofold: (i) to investigate formation and transformation of $\mathrm{CaP}$ minerals within a synthetic hydrogel matrix with molecular architecture, and (ii) to assess the effect of citrate and zinc to stabilise ACP in such matrices. Mineralised hydrogel composites were formed in a simple one-step process by mixing two aqueous components composed of a polymer together with phosphate ions, and an end-linking peptide together with calcium ions. Optionally, citrate or zinc was added to stabilise ACP. Upon mixing, the system simultaneously gelled and mineralised. To study CaP precipitation and transformation within the hydrogel 
matrix, a comprehensive set of complementary and advanced methods was used in wet and dry state.

\section{Experimental}

\subsection{Reagents}

Eight-armed maleimide-functionalised polyethylene glycol (PEG) macromeres with an average molecular weight of $40 \mathrm{kDa}$ were purchased (40M8; JenKem Technologies, USA). Degree of functionalisation and purity were determined to be $99.4 \%$ and $100 \%$ by ${ }^{1} \mathrm{H}-\mathrm{NMR}$ and HPLC, respectively, by the manufacturer. Enzymatically cleavable end-linking peptide was synthesised as acetate salt (XLP, sequence Ac-GCRDVPMSMRGGDRCG-NH 2 [40]; GenScript, USA). Net peptide concentration was determined by the supplier via $\mathrm{C} / \mathrm{H} / \mathrm{N}$ element analysis to be $85.6 \%$ and peptide purity via HPLC to be $95.1 \%$. Zinc nitrate hexahydrate was purchased from Thermo Fisher (Germany). All other reagents were obtained from SigmaAldrich (Norway). Deionised water with a resistivity $>18 \mathrm{M} \Omega$ was used in all experiments.

\subsection{Composite preparation}

Mineralised hydrogel composites were produced by mixing two precursor components. Component A contained 40M8 in a mixture of mono- and dibasic ammonium phosphate at $\mathrm{pH}$ 5.5. In component $\mathrm{B}$, an amount of end-linking peptide stoichiometrically matched to the concentration of maleimide groups in component A was mixed with a calcium nitrate solution. For citrate-containing composites, a mixture of dibasic ammonium citrate and citric acid of pH 5.5 was included in component A. For zinc-substituted samples, calcium nitrate in component B was partially replaced with zinc nitrate. Exact formulations of the different groups are given in Table 1. 
Table 1: Chemical composition of CaP-hydrogel composites without additives (NoAdd), with $50 \mathrm{mM}$ citrate (Cit50), and with $10 \%$ zinc substitution (Zn10). Presented concentrations refer to the total mixture of A and B.

\begin{tabular}{|c|c|c|c|c|c|c|}
\hline \multirow[t]{3}{*}{ Sample } & \multicolumn{3}{|c|}{ Component A } & \multicolumn{3}{|c|}{ Component B } \\
\hline & $40 \mathrm{M8}$ & Amm. phosph. pH 5.5 & Amm. cit. pH 5.5 & End-linker & $\mathrm{Ca}\left(\mathrm{NO}_{3}\right)_{2}$ & $\mathrm{Zn}\left(\mathrm{NO}_{3}\right)_{2}$ \\
\hline & $(w t . \%)$ & $(\mathbf{m M})$ & $(\mathbf{m M})$ & $(\mathbf{m M})$ & $(\mathbf{m M})$ & $(\mathbf{m M})$ \\
\hline NoAdd & 5 & 200 & 0 & 5 & 400 & 0 \\
\hline Cit50 & 5 & 200 & 50 & 5 & 400 & 0 \\
\hline Zn10 & 5 & 200 & 0 & 5 & 360 & 40 \\
\hline
\end{tabular}

Gelation and simultaneous mineralisation were initiated by mixing components $\mathrm{A}$ and $\mathrm{B}$. For in situ observations, approximately $2 \mu 1$ of the mixture was placed in a simple flow cell as previously reported [41] (Figure 1a). For ageing experiments, larger disk-shaped samples were produced as described previously [42] (Figure 1b). In brief, $20 \mu 1$ of the mixture was sandwiched between two silanised glass slides separated by a $1.5 \mathrm{~mm}$ thick spacer. In both setups, the formed composites were allowed to cure for five minutes before being flushed with 100 mM Tris-HCl buffer $\mathrm{pH}$ 7.4. 
a)

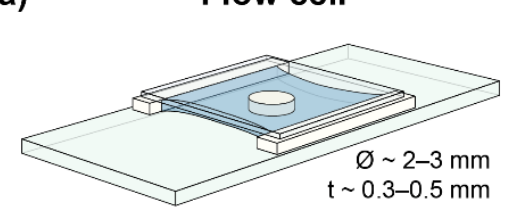

b)

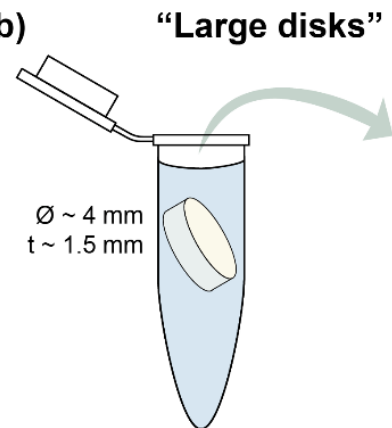

In situ observation:

- DFLM

- CLSM (pH mapping)

- Raman (fresh)

Figure 1: Characterisation experiments were performed a) in situ on samples mounted in a flow cell and b) on the surface or cross-section of disk-shaped samples that have been aged in buffer.

\subsection{Mineral characterisation}

\subsubsection{Dark-field microscopy}

Distribution and morphology of precipitating mineral was observed in a flow cell (Figure 1a) by dark-field microscopy (DFLM; Eclipse TS100, Nikon, Japan) equipped with a 4x objective. Images were captured with a sCMOS camera (Zyla 5.5, ANDOR, UK) at $0.1 \mathrm{fps}$.

\subsection{2 pH monitoring}

$\mathrm{pH}$ plays a pivotal role in the mineralisation of the hydrogel composites. The $\mathrm{pH}$ in mineralising composites was spatiotemporally measured using a modified technique reported elsewhere [41]. $5 \mu \mathrm{M}$ pH-sensitive R6G-EDA dye (ethylenediamine modified rhodamine 6G [43]) and $2 \mu \mathrm{M}$ pH-insensitive ATTO 643 dye (ATTO-TEC, Germany) were added to both precursor components and the flushing buffer. Composites in a flow cell were imaged using a confocal laser-scanning microscope (CLSM; TCS SP8, Leica Microsystems, Germany) with a Leica HCX PL FLUOTAR 5x/0.15 NA objective at 0.1 fps. Two 488 and $638 \mathrm{~nm}$ diode-pumped solid-state lasers were scanned sequentially across the sample line-by-line, exciting $\mathrm{pH}$ - 
sensitive and -insensitive dyes, respectively. After applying a $3 \times 3$ median filter on the recorded images, the ratio of the R6G-EDA and the ATTO 643 images was used to calculate a pH value for each pixel using MATLAB R2017a (MathWorks, USA). A calibration curve was obtained using buffered standards of known $\mathrm{pH}$ (citrate-phosphate buffer for $\mathrm{pH} 3.5$ to 6.5 and Tris-HCl buffer for $\mathrm{pH} 7.0$ to 8.0 in increments of $0.5, \mathrm{n}=3$ ). The R6G-EDA to ATTO 643 fluorescence ratio was used for $\mathrm{pH}$ values below 5.5, and the inverse for $\mathrm{pH}$ values above 5.5.

\subsubsection{Scanning electron microscopy}

$\mathrm{CaP}$ phases were qualitatively characterised based on mineral morphology. Morphology of mineral particles was analysed using scanning electron microscopy (S-4800, Hitachi, Japan). Prior to SEM analysis, composites were immersed in deionised water for one minute to remove non-precipitated ions, cut with a scalpel, snap-frozen in liquid nitrogen, and lyophilised for $48 \mathrm{~h}$ (LyoQuest -85, Telstar Life Science Solutions, Spain). Dried samples were sputter coated with 10 nm Pt (208 HR, Cressington Scientific Instruments, UK). Particle sizes were estimated from SEM images using the open source software Fiji [44].

\subsubsection{Atomic absorption spectroscopy}

The amounts of calcium and zinc in different composites were determined using atomic absorption spectroscopy (AAS; AANALYST 400, Perkin Elmer, USA). AAS samples were acidified with $\mathrm{HCl}$ to dissolve precipitated mineral before lanthanum chloride was added at a concentration of $10 \mathrm{mg} / \mathrm{mL}$ to precipitate phosphate. Acetylene was used as carrier gas. Three independent samples were measured per data point.

\subsubsection{Spectroscopy}

Raman and Fourier transformed infrared spectroscopy (FTIR) was employed to characterise the mineral phase. Confocal Raman micro-spectroscopy (CRM) measurements were performed using an InVia Reflex system (Renishaw, UK) with a $532 \mathrm{~nm}$ laser through a Leica 10x/0.25NA objective. For early observations, samples were mounted in a flow cell (Figure 1a) of Raman- 
grade $\mathrm{CaF}_{2}$ slides (Crystran, UK). Cross-sections of aged composite disks were analysed on the same substrate. The spot size of the laser probe was approximately $15 \mu \mathrm{m}$ and $3 \mathrm{scans}$ of $30 \mathrm{~s}$ were accumulated per spectrum. For thermal annealing experiments, disk samples were dried and thermally annealed. First, disks were briefly washed in deionised water to remove nonprecipitated ions and consecutively dehydrated using ethanol series, acetone, and critical point drying (Emitech K850, Quorum Technologies, UK). Dried samples were heat-treated in a furnace (AAF 1100, Carbolite Gero, UK) for $8 \mathrm{~h}$ at $700^{\circ} \mathrm{C}$ to crystallise potential ACP.

FTIR analysis was performed on a Spectrum 400 spectrometer (Perkin Elmer, USA) with a diamond/ZnSe universal attenuated total reflection (ATR) module. Composite samples for FTIR analysis were produced as disks (Figure 1b). Scans were performed at a resolution of $2 \mathrm{~cm}^{-1}$ and 16 scans were accumulated per measurement.

\subsubsection{Scanning precession electron diffraction}

To characterise the mineral phase on a nanometre scale, a TEM specimen of a three day aged citrate-stabilised composite was produced as follows: aqueous solvent was replaced using ethanol series, followed by acetone, and eventually epoxy resin (hardener MNA \& DDSA with accelerator DMP, Sigma-Aldrich, Norway). The resin was cured at $60^{\circ} \mathrm{C}$ for three days. Thin sections $(80 \mathrm{~nm})$ were cut from the resin block using a microtome (Ultracut $\mathrm{S}$, Reichert, USA) equipped with a with a diamond knife (ultra $45^{\circ}$, DiATOME, USA) and placed on Cu grids with a lacey carbon film (300 mesh, Agar Scientific, UK).

Scanning precession electron diffraction (SPED) scans were performed on a JEM-2100F (FEG) TEM (JEOL, Japan) operated at $200 \mathrm{kV}$, equipped with an ASTAR system (Nanomegas, Belgium) for SPED acquisition. The electron beam was scanned over the specimen area at $1.28 \mathrm{~nm}$ step size with a precession angle of $1^{\circ}$ and a dwell time of $50 \mathrm{~ms}$ per pixel. The camera length of the system was $40 \mathrm{~cm}$, giving a theoretical calibration constant of $0.16(\mathrm{~nm} \cdot \mathrm{px})^{-1}$. Diffraction patterns (DP) were recorded from the viewing screen using a Stingray camera 
(144x144 pixels, 8 bits). Standard bright-field TEM images were obtained using the same instrument.

The open source software Hyperspy 1.3.1 [45] was used to analyse the multidimensional SPED datasets. Virtual bright- and dark-field images were reconstructed from the electron diffraction patterns using virtual apertures. DPs were compared to kinematic simulations (CrysTBox Server 1.102 [46]; COD IDs 2300273, 2310526, and 1534327 for HA, dicalcium phosphate dihydrate (brushite, DCPD), and octacalcium phosphate (OCP), respectively; Appendix 5). In order to map sample regions with principally different DPs, a circular virtual aperture with a radius of $0.2 \mathrm{~nm}^{-1}$ was incrementally moved along a circular path of radius $d_{h k l}{ }^{-1}$ around the central beam (see provided animations and Appendix 6). Intensity maps were reconstructed from the aperture positions that yield maximal intensities. In a further step, reflections originating from hexagonal DPs were separated from non-hexagonal patterns with the $d$ spacing. This was achieved by considering intensities of any given aperture position $(\varphi)$ and the corresponding positions for a hexagonal pattern $\left(\varphi+60^{\circ}\right.$ and $\left.\varphi+120^{\circ}\right)$. The reconstructions from the hexagonal aperture arrangement yielding the highest intensity for the current pixel were thresholded against the average intensity of all aperture positions $\varphi$ for the same pixel.

\subsection{Statistical analysis}

Results of the cation release measurements were statistically analysed. Normality of sample distribution was tested using the Shapiro-Wilk test, followed by testing statistical significance using one-way analysis of variance (SigmaPlot 13.0, Systat Software, USA). A p-value $\leq 0.05$ was considered significant. 


\section{Results}

\subsection{Mineral precipitation and morphology}

Mineralised CaP-hydrogel composites were produced in a simple one-step procedure. To investigate $\mathrm{CaP}$ precipitation within the hydrogel matrix, mineral-free gels (Plain), composites containing only gel precursors, calcium, and phosphate (NoAdd), and composites containing citrate or zinc additives (Cit50 and Zn10) were prepared as described in section 2.2. Upon mixing of the two precursor components, mineral precipitated simultaneously to gelation of the polymeric matrix. Five minutes after mixing, samples were flushed with buffer to mimic injection into a bone defect.

SEM images of composites ten minutes after flushing show mineral particles of uniform, particulate morphology typical for ACP in all mineralised samples (insets in Figure 2d-f). Particle sizes were estimated from the micrographs to be $230 \pm 30,160 \pm 40$, and $190 \pm 60 \mathrm{~nm}$ (mean \pm standard deviation, 30 random particles measured per group) for NoAdd, Cit50, and Zn10 composites, respectively. Only in NoAdd samples, we also found a number of large crystals featuring a rhombic tabular morphology specific for brushite (Figure 2b-d). Interestingly, these crystals almost exclusively occurred on the composite surface. Very few crystals were found inside the hydrogel matrix (Figure $2 \mathrm{~g}$ ). The evolution of the observed mineral particles was visualised by DFLM. Since both $\mathrm{CaP}$ precipitation and gelation are $\mathrm{pH}-$ dependent processes, $\mathrm{pH}$ was spatiotemporally monitored on separate samples using a $\mathrm{pH}$ sensitive CLSM setup. 

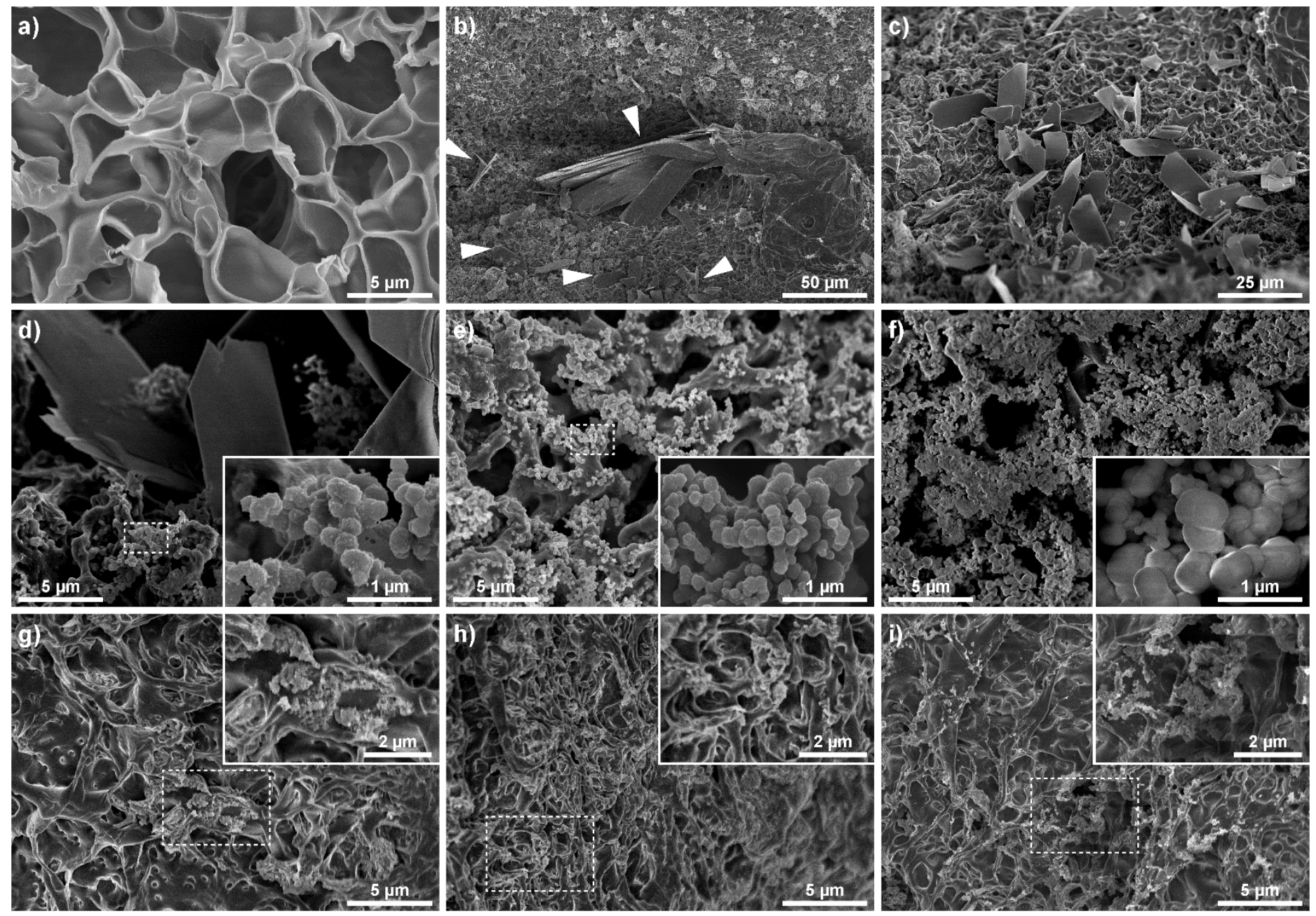

Figure 2: SEM images of dehydrated composites aged for 10 minutes in Tris- $\mathrm{HCl}$ buffer at $\mathrm{pH} 7.4$ after the mineralisation step was stopped by flushing. a) Non-mineralised gel matrix for reference. b-f) Surface views of NoAdd (b-d), Cit50 (e), and Zn10 (f) composites show characteristic brushite crystals (arrowheads in b) co-existing with the amorphous mineral particles in the additive-free samples and only amorphous mineral particles in the samples containing citrate or zinc. g-i) Cross-section views of NoAdd (g), Cit50 (h), and Zn10 (i) samples. Insets show magnifications of clusters of spherical mineral particles.

Small precipitated mineral particles were present throughout all $\mathrm{CaP}$-containing samples from the very beginning of the microscopic observation (Figure 3), showing that initial mineral precipitation occurred within 30 to $60 \mathrm{~s}$. While the initial precipitation occurred too quickly to be captured by DFLM, it was evident as an increase in turbidity of the initially clear precursor mix during sample handling. Qualitative differences were observed for the individual sample 
groups. Precipitation occurred almost instantaneously in Zn10 samples, followed by NoAdd and Cit50 samples, the latter turning turbid at a much slower rate. The differences in precipitation rate affected the homogeneity of the initial precipitate as seen in DFLM (Figure 3e,i,m). At the moment of sample mounting, a continuous network of mineral particles had already formed in Zn10 and NoAdd composites. Sandwiching the viscous sample droplet between two glass slides, this mineral network was disrupted, resulting in a slightly inhomogeneous mineral distribution. In Cit50 samples, on the other hand, no continuous mineral network had formed yet at the time point of mounting, explaining the very homogeneous distribution of mineral throughout the sandwiched sample.

The large brushite crystals besides the initial precipitate identified in SEM are also detectable in low magnification DFLM of NoAdd composites (arrowheads in Figure 3e-g). The crystals were present from the very beginning of the microscopic observation and continuously grew in size over the shown observation time, consuming the homogeneous initial phase around them. In Cit50 and Zn10 composites, no crystals or dissolution of the initial precipitate was observed within one hour after mixing (animations provided in Appendix). 

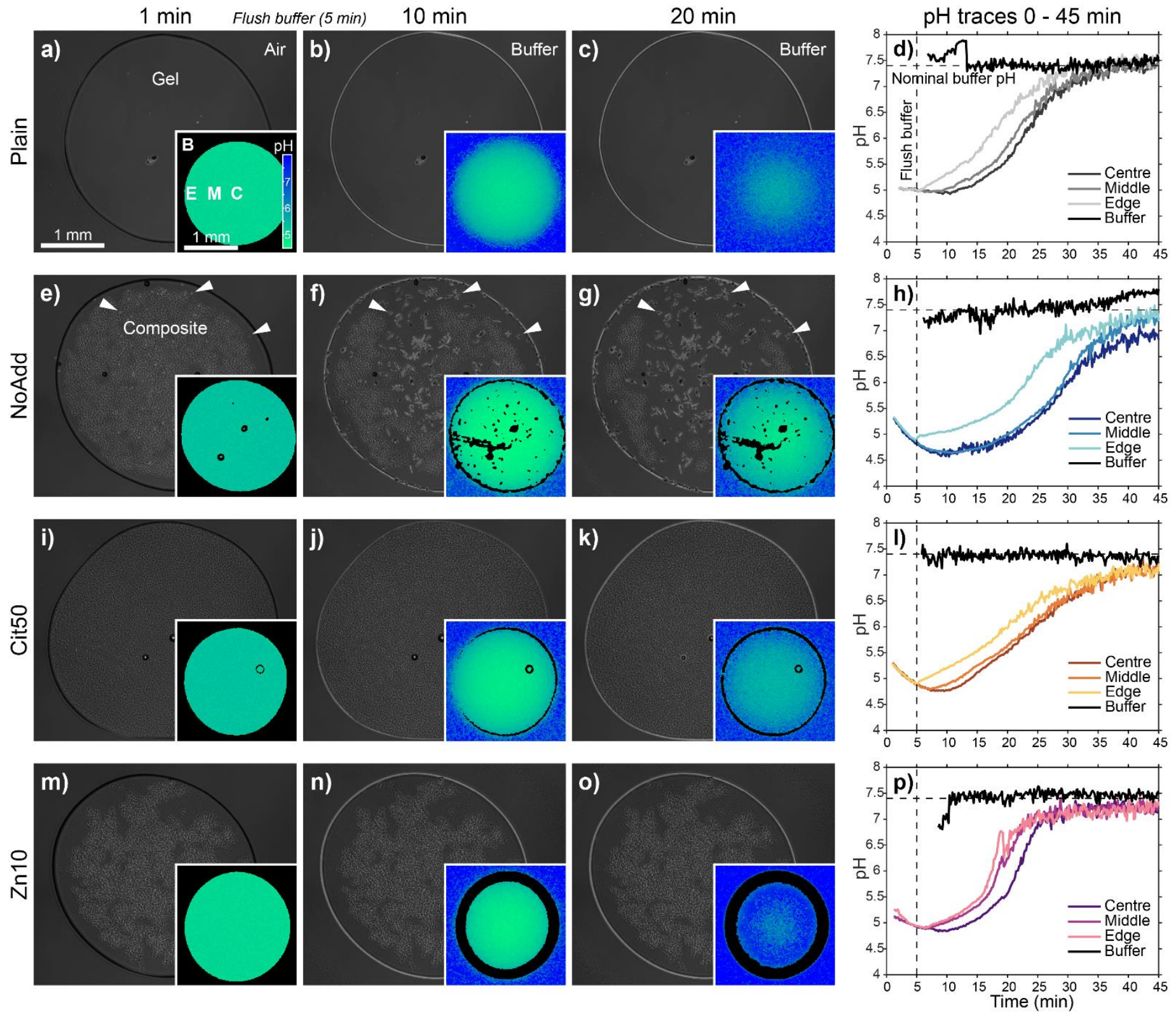

Figure 3: a-c, e-g, i-k, m-o) Dark-field micrographs of mineral-free (a-c) and mineralised hydrogel-CaP composites without additives (e-g), with added citrate (i-k), or zinc (m-o). Pseudocolour insets show pH maps of equivalent samples as determined by CLSM using a pH-sensitive dye arrangement. Five minutes after mixing, samples were flushed with Tris- $\mathrm{HCl}$ buffer. $\mathrm{d}, \mathrm{h}, \mathrm{l}, \mathrm{p}$ ) Traces of the $\mathrm{pH}$ values over time are shown for regions of interest in the buffer (B), close to the composite edge (E), halfway into the sample (M), and at the centre (C). Arrowheads indicate selected crystals. Animations of the above data are available as supplementary material.

Following flushing, additional mineral precipitated at the composite-buffer interface. SEM images of composites dehydrated ten minutes after flushing show a dense layer of mineral on 
the surface (Figure 2d-f) and considerably less mineral inside the composites (Figure $2 \mathrm{~g}$-i). The densely mineralised shell is also visible in pseudocolour $\mathrm{pH}$ maps (insets in Figure 3 ) where these regions appear black, as dense mineral agglomerates mask out a substantial proportion of the fluorescent signal. $\mathrm{pH}$ traces for small regions of interest close to the edge, middle, or centre of the composites provide additional information about mineral formation in different composites.

$\mathrm{pH}$ monitoring revealed that the different composites underwent different $\mathrm{pH}$ changes (Figure $3 \mathrm{~d}, \mathrm{~h}, 1, \mathrm{p})$. In the absence of mineral, the $\mathrm{pH}$ inside the hydrogel gradually increased from the initial pH 5.0 to 7.4, as flushing buffer diffused into and reaction buffer out of the matrix. After $\sim 35$ minutes, the entire gel reached $\mathrm{pH}$ 7.4. Within the composites, on the other hand, the $\mathrm{pH}$ initially dropped from 5.5 at mixing to approximately 4.7 as $\mathrm{H}^{+}$was released from $\mathrm{H}_{2} \mathrm{PO}_{4}{ }^{-}$and $\mathrm{HPO}_{4}{ }^{2-}$ during precipitation. While the $\mathrm{pH}$ traces of the different composite groups show the same general pattern, they revealed some differences between the distinct groups. For NoAdd and Cit50 composites, the $\mathrm{pH}$ in the centre of the samples kept falling until about seven and five minutes after flushing, respectively, indicating that mineral continued to precipitate. Thereafter, the $\mathrm{pH}$ inside the samples slowly increased towards buffer level, which was reached after about 40 to 45 minutes. Zn10 samples differ from this; at the beginning of the measurement, $\sim 30 \mathrm{~s}$ after mixing, the $\mathrm{pH}$ had already fallen to 5.1, approximately $0.3 \mathrm{pH}$ units lower compared to NoAdd or Cit50 composites. This is a consequence of the notably faster initial precipitation observed in Zn10 composites. After flushing, Zn10 samples were readily buffered to $\mathrm{pH} 7.4$, indicating that little further precipitation occurred.

The effect of ageing was investigated on larger disk-shaped samples (Figure 1) immersed in Tris-HCl buffer. The mineral content throughout ageing was determined by AAS. Figure 4 shows that calcium and zinc were released in two stages: a high initial release was followed by a steady release at a slow rate. For all groups, about half of the total calcium was released into 
the buffer after ten minutes. Over three days of ageing, the calcium concentration decreased by another $\sim 15 \%$ of the initial amount, indicating slow mineral dissolution. While the differences in the calcium content are not statistically significant for early time points, we found that after three days, NoAdd and Zn10 composites contained significantly less calcium than Cit50 samples. Remarkably, after ten minutes, only $8.2 \%$ of the total zinc content was released from $\mathrm{Zn} 10$ composites compared to $56.7 \%$ of the total calcium. This indicates that the majority of zinc ions precipitated within the composite while a large proportion of calcium ions remained in solution. Following the initial release, relative zinc and calcium release proceeded at comparable rates. Approximately $25 \%$ of the remaining calcium and zinc were released over three days.

a)

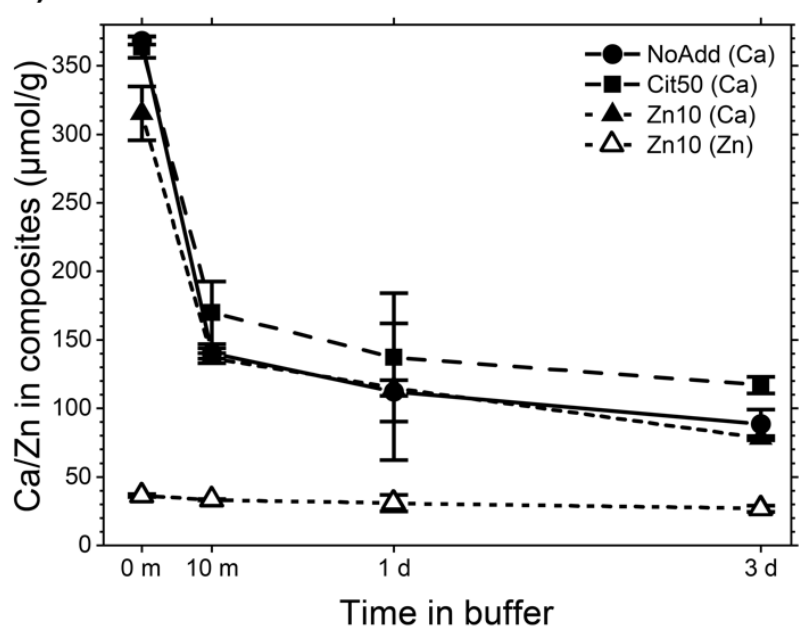

b)

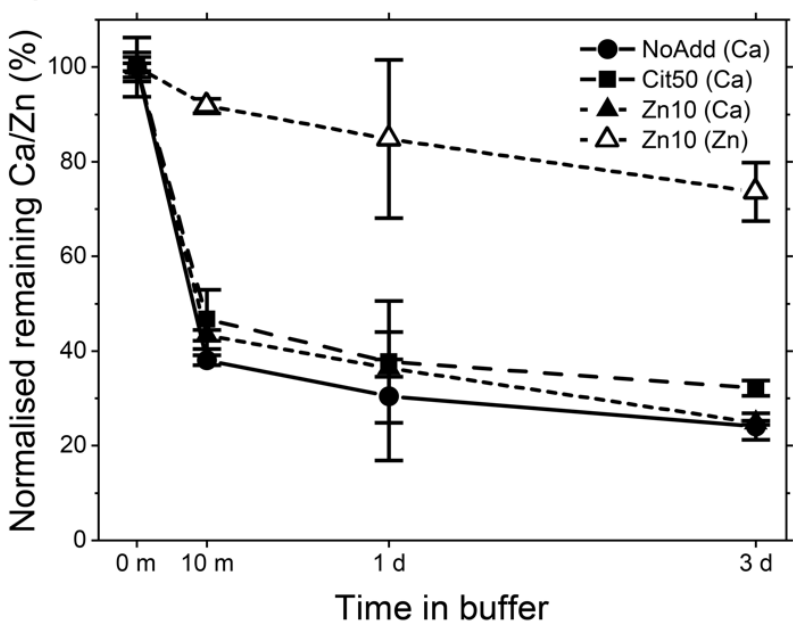

Figure 4: a) Absolute and b) normalised cation concentrations for composites aged in buffer. The amounts of calcium (filled symbols) and zinc (open symbols) that remained within the composites during ageing were determined by AAS. In b), values are normalised to the measured concentrations before flushing (0 minutes). Markers and error bars represent mean values and standard deviation $(n=3)$.

SEM images of samples aged three days in buffer reveal that the different composite groups were affected differently by ageing (Figure 5). In $\mathrm{Zn} 10$ composites, the mineral distribution and general morphology had not changed much compared to ten minutes after flushing (Figure $2 \mathrm{f} \& \mathrm{i}$ vs. Figure 5c\&f). Clusters of mineral were still present throughout the entire sample. Only the 
roughness of the particles had increased notably, indicating surface erosion during ageing. In NoAdd and Cit50 samples, on the other hand, particle morphology had completely changed from particulate spheres to larger rosettes of entangled plate-like crystals (insets in Figure $5 \mathrm{a} \& \mathrm{~b})$. The surface of Cit50 samples was homogeneously covered by a layer of mineral (Figure 5b) and a few crystal clusters were spotted deeper in the sample (arrowheads in Figure 5e). In NoAdd samples, in contrast, mineral was only found in dense isolated patches on the sample surface (Figure 5a). No mineral was found within the hydrogel matrix (Figure 5d). The architectures of the hydrogel matrices were comparable for all composite groups, exhibiting similar microporous structures as observed in non-mineralised hydrogels (Figure 2a). This indicates that the mineralisation process did not notably affect the hydrogel formation.
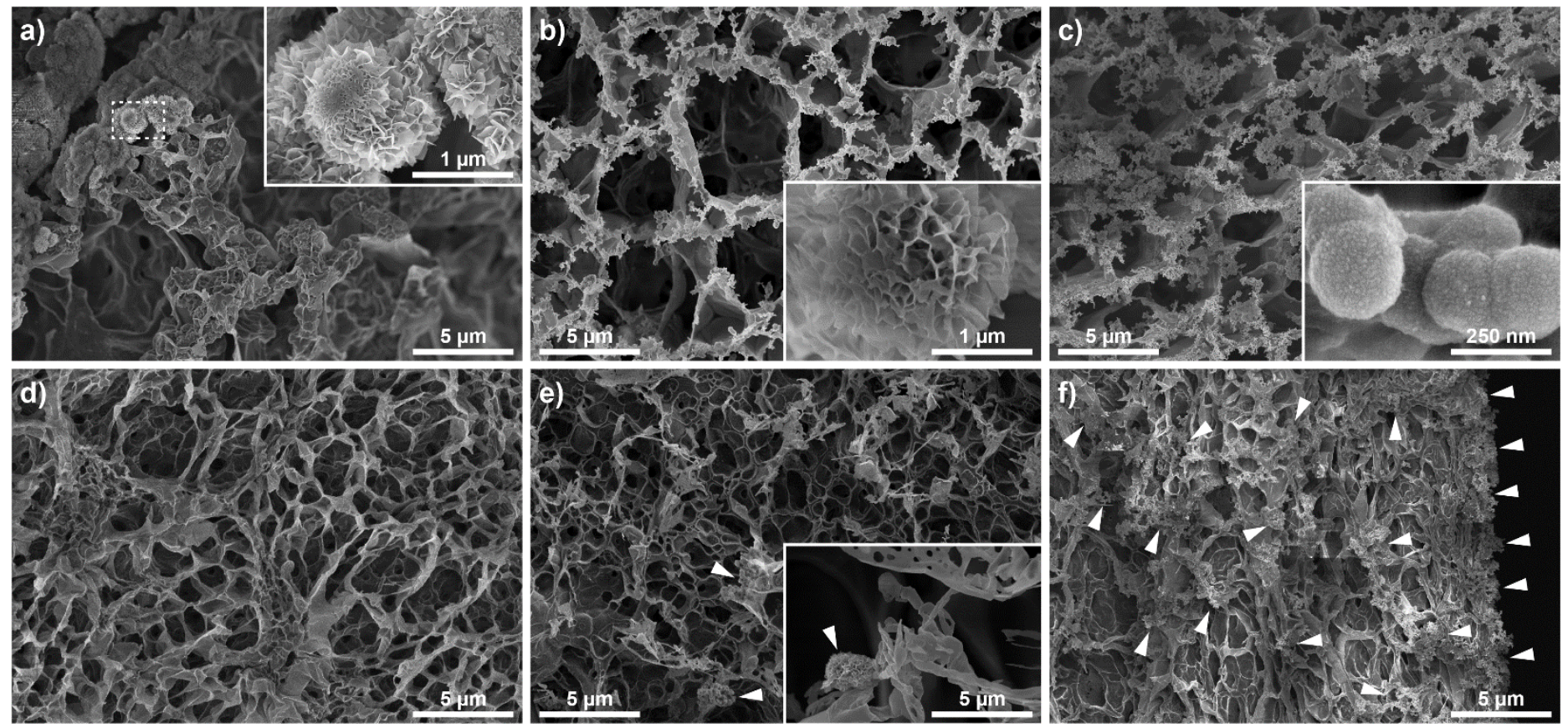

Figure 5: SEM images of dehydrated composites aged three days in Tris-HCl buffer of $\mathrm{pH}$ 7.4. a-c) Surface views of NoAdd (a), Cit50 (b), and Zn10 (c) composites show entangled plate-like CaP crystals in the additive-free and the citrate-containing group and spherical amorphous particles in the zinc-containing group. d-f) Cross-section views of NoAdd (d), Cit50 (e), and Zn10 (f) show little mineral inside the samples for the additive-free and citratecontaining group, while clusters of mineral particles (arrowheads) are evenly distributed throughout the zinccontaining composites. Insets depict magnifications of mineral particles. 


\subsection{Mineral phase characterisation}

To determine the phase composition of the mineral precipitated in the hydrogel matrices, hydrated composites were analysed using confocal Raman micro-spectroscopy (CRM) and ATR-FTIR. For early time points, CRM analysis was performed on composite disks mounted in a $\mathrm{CaF}_{2}$ flow cell (Figure 1). For later time points, larger composite disks were aged in buffer. Cross-sections were cut with a scalpel and mounted between $\mathrm{CaF}_{2}$ substrates. ATR-FTIR analysis on composite disks aged in buffer was used to complement Raman results, examining mineral close to the composite surface.

Approximately ten minutes after flushing, background-subtracted Raman spectra for bulk composites revealed no substantial differences depending on sample group or location in the composite (solid traces in Figure 6a; also refer to Figure A 1 for spectra of the individual hydrogel components). The spectra show no peaks apart from those of the hydrogel matrix, indicating the absence of crystalline phases in the bulk composites. However, when focusing on one of the individual crystals found in NoAdd composites, a different spectrum was obtained (dashed trace in Figure 6a). Distinct peaks at $985\left(v_{1} \mathrm{HPO}_{4}{ }^{2-}\right), 877(\mathrm{vPOH})$, and $524-586 \mathrm{~cm}^{-1}$ $\left(v_{4} \mathrm{HPO}_{4}{ }^{2-}\right)$ were assigned to DCPD [22] and confirm our earlier assumption based on crystal morphology. 
a)

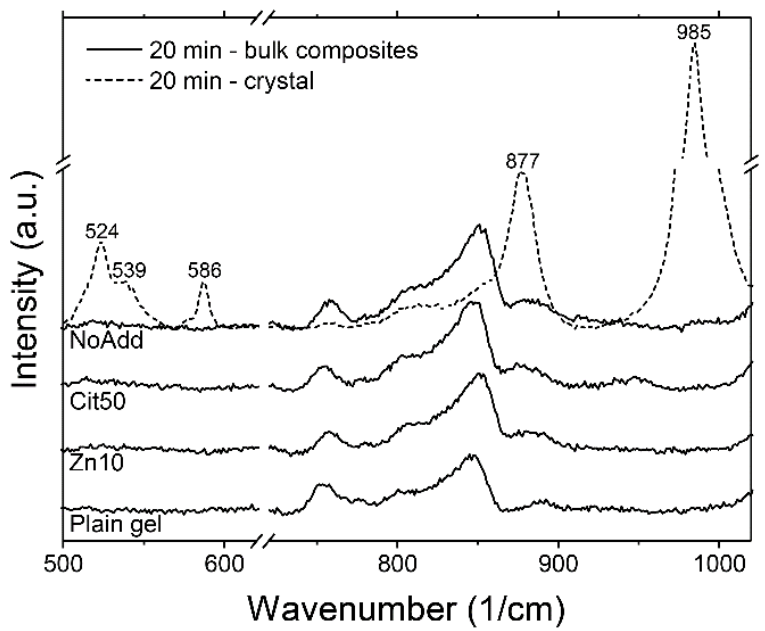

c)

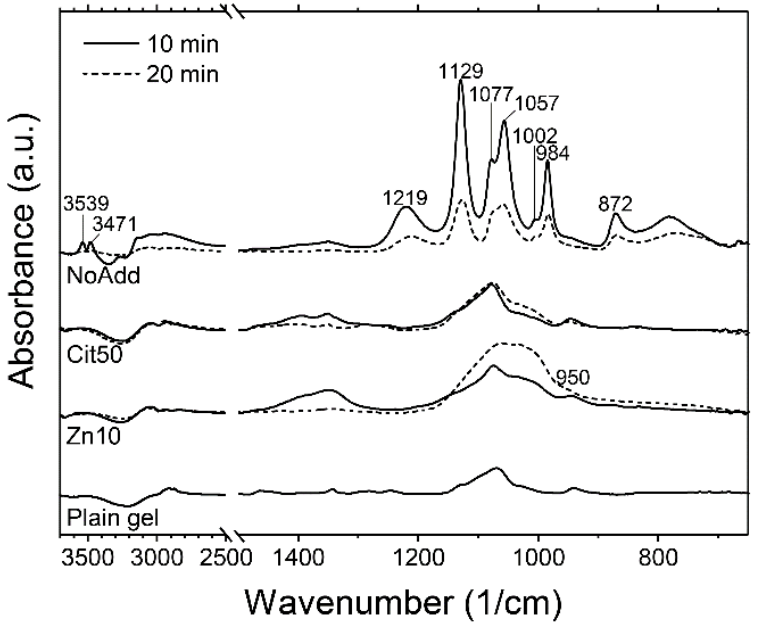

b)

Raman, aged samples

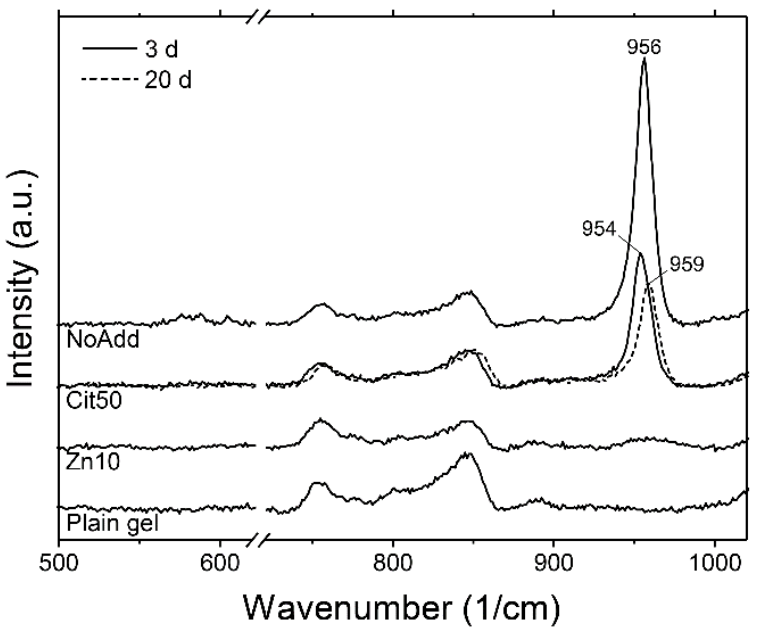

d)

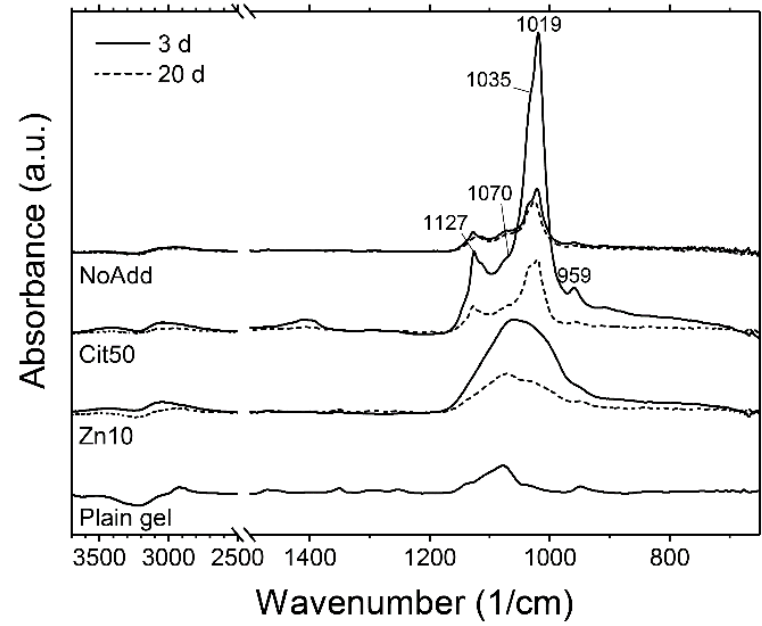

Figure 6: Raman and FTIR spectra of composites 10 to 20 minutes after flushing with Tris-HCl buffer of pH 7.4 and after 3 and 20 days of ageing. a) Raman spectra obtained on bulk composites 20 minutes after flushing (solid lines) show no distinct peaks other than the ones associated to the plain hydrogel. A spectrum obtained on a large crystal found in NoAdd composites (dashed line in a) matches brushite. b) Raman spectra of samples aged 3 and 20 days were obtained from the densely mineralised region close to the sample surface and match with OCP or HA in NoAdd and Cit50 samples, while Zn10 samples show no peaks indicating crystalline CaP. c) ATR-FTIR absorbance spectra of composite surfaces 10 and 20 minutes after flushing confirm the observations from Raman. The spectra of citrate- and zinc-containing composites additionally reveal a broad peak from $\sim 900-1150 \mathrm{~cm}^{-1}$ indicating ACP. d) ATR-FTIR spectra of samples after 3 and 20 days of ageing in buffer match with OCP or HA for NoAdd and Cit50 samples and ACP for Zn10 samples. All graphs show spectra of non-mineralised gels for reference. 
FTIR scans of samples aged for 10 and 20 minutes in buffer confirmed the findings from CRM (Figure 6c). A strong DCPD signal originating from the brushite crystals on the sample surface was observed for the NoAdd samples [47]. Between 10 and 20 minutes, the DCPD signal decreased in intensity as brushite crystals detached from the surface of the samples immersed in buffer. After ten minutes in buffer, Zn10 samples exhibited only a weak continuous absorbance band from $\sim 900-1150 \mathrm{~cm}^{-1}$ with a $v_{1}\left(\mathrm{PO}_{4}\right)$ shoulder at $\sim 950 \mathrm{~cm}^{-1}$. After 20 minutes, this spectrum developed into a broad, featureless peak characteristic of an isotropic amorphous phase [47]. No prominent CaP modes were detected for Cit50 10 and 20 minutes after flushing. Subtraction of the hydrogel signal, however, revealed a weak absorbance band from 900 $1150 \mathrm{~cm}^{-1}$ that was qualitatively similar to the one observed for $\mathrm{Zn} 10$, indicating ACP (Figure A 2). Further indirect evidence for the amorphous nature of the mineral was provided in a thermal annealing experiment. Raman spectra obtained at the different stages of the treatment clearly revealed temperature-induced crystallisation (Figure 7). While we found a $v_{1}\left(\mathrm{PO}_{4}\right)$ peak at $960 \mathrm{~cm}^{-1}$ and $v_{4}\left(\mathrm{PO}_{4}\right)$ peaks at $580-590 \mathrm{~cm}^{-1}$ assigned to apatite for the thermally annealed sample, no crystalline phase was evident in the wet or dried composite. This implies that the mineral must have been amorphous prior to annealing. 


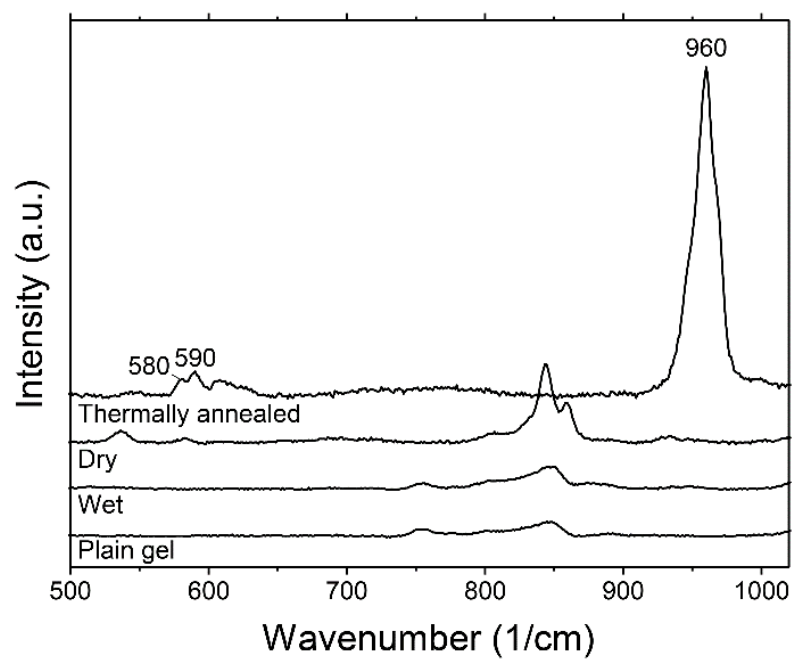

Figure 7: Raman spectra for a Cit50 composite after thermal annealing. A Cit50 composite was aged for $10 \mathrm{~min}$ in buffer, dehydrated, and heat-treated for $8 \mathrm{~h}$ at $700^{\circ} \mathrm{C}$. While the spectrum of the sample after heat treatment shows a distinct peak at $960 \mathrm{~cm}^{-1}$, no indication of a crystalline CaP phase was found before heat treatment, which indicates that the CaP mineral is amorphous. The spectrum of a non-mineralised gel is shown for reference.

Mineral transformations occurring during ageing were evident in Raman and FTIR spectra of samples stored three days in buffer. CRM spectra obtained close to the edge of cross-sectioned samples show clear evidence of crystalline CaP for NoAdd and Cit50 samples (Figure 6b). The peaks at 956 and $954 \mathrm{~cm}^{-1}$ for NoAdd and Cit50, respectively, correspond to $v_{1} \mathrm{PO}_{4}{ }^{3-}$ stretching vibrations occurring in OCP and HA. Over extended ageing of Cit50, this peak was slightly shifted from 954 to $959 \mathrm{~cm}^{-1}$, indicating an ongoing transformation. Similar to Raman, ATRFTIR scans of NoAdd and Cit50 samples revealed spectra that can be assigned to OCP or poorly crystalline HA (Figure 6d). While the spectra of both samples are qualitatively very similar, it is notable that the Cit50 spectrum is of stronger intensity, suggesting more crystalline mineral on the composite surface, as also seen in SEM (Figure 5a\&b). Zn10 samples still showed a pronounced ACP peak in FTIR and no evidence of a crystalline phase in Raman. However, between 3 and 20 days in buffer, the intensity of the absorption band in FTIR decreased, suggesting that mineral slowly dissolved. 
Cross-sections of three-day aged samples scanned in CRM revealed significant spatial inhomogeneity (see Figure 8). As observed in SEM, NoAdd and Cit50 samples exhibited a densely mineralised shell and little mineral in the centre of the sample, whereas Zn10 samples featured a relatively homogenous distribution of mineral. Similarly, Raman intensities for the peaks at 954 and $956 \mathrm{~cm}^{-1}$ decreased with distance from the sample edge. Figure 8 further reveals that throughout the mineralised regime, this peak was shifted to higher wavenumbers for the NoAdd sample compared to Cit50, indicating a more HA-like phase in the former and a more OCP-like phase in the latter sample.

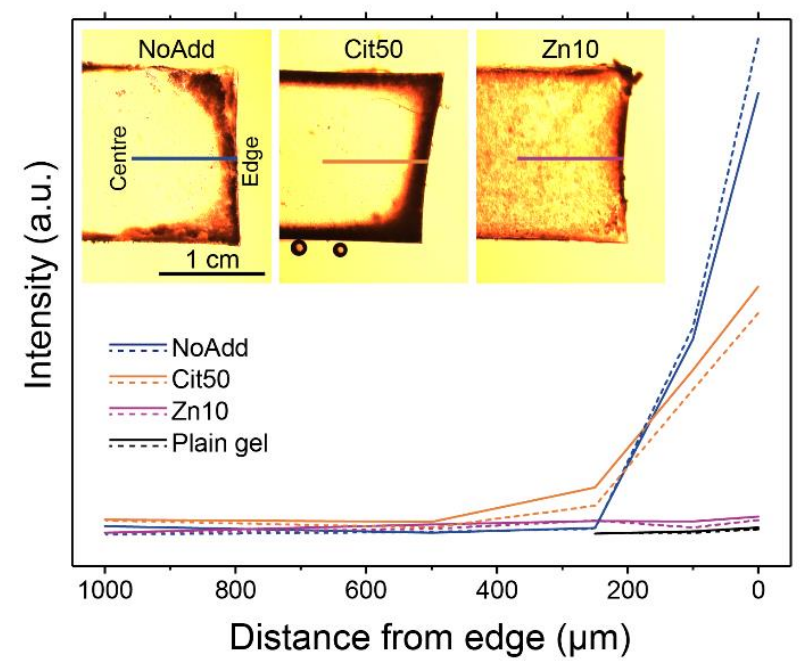

Figure 8: Raman intensities for line scans along the cross-sectional surface of different composites (see lines in inset light micrographs) and a non-mineralised gel aged three days in buffer. Intensity plots for the peaks at $954 \mathrm{~cm}^{-1}$ (solid traces) and $956 \mathrm{~cm}^{-1}$ (dashed traces) reveal that crystalline CaP mineral is only found close to the composite surface for NoAdd and Cit50 samples.

Due to their very similar spectra, it is difficult to differentiate OCP and low crystalline HA based purely on spectroscopic data or morphology. To clarify the identity of the mineral phase in three-day aged Cit50 composites, a SPED experiment was performed. In SPED, a precessing electron beam is scanned across a TEM sample collecting a DP for every pixel in the image 
[48]. These DPs appear more kinematic than static (i.e. not precessed). They can be used to reconstruct various images and to map crystal phases and orientation.

Bright-field TEM of the scanned Cit50 composite revealed clusters of nano-sized plate-like particles (see Figure 9a). The indicated area was analysed via SPED. A virtual bright-field reconstruction, accounting for reflections covering the scattering range from $0.2-1 \mathrm{~nm}^{-1}$, revealed the background signal originating from potentially present amorphous mineral, polymeric network, and epoxy resin (Figure 9b). Strongly diffracting particles appear dark and coincide with the particles observed in the TEM image. A virtual dark-field reconstruction of the same dataset, covering the scattering range between $5-10 \mathrm{~nm}^{-1}$, revealed similar features in positive contrast (Figure 9c).

TEM BF

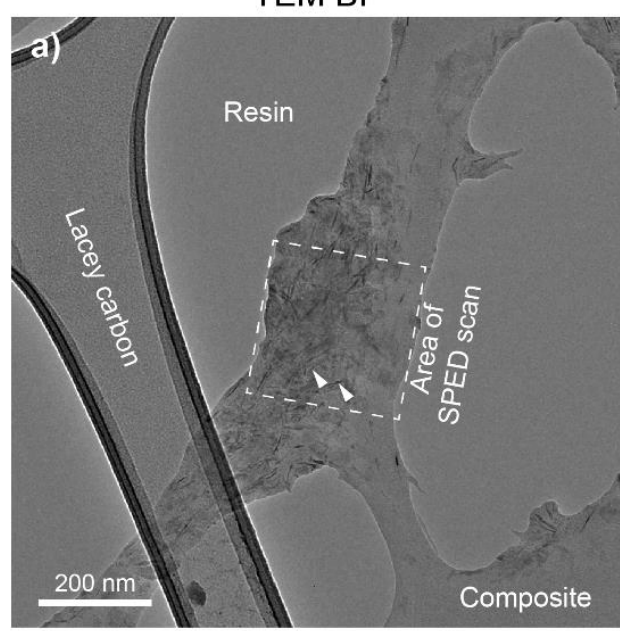

SPED virtual BF

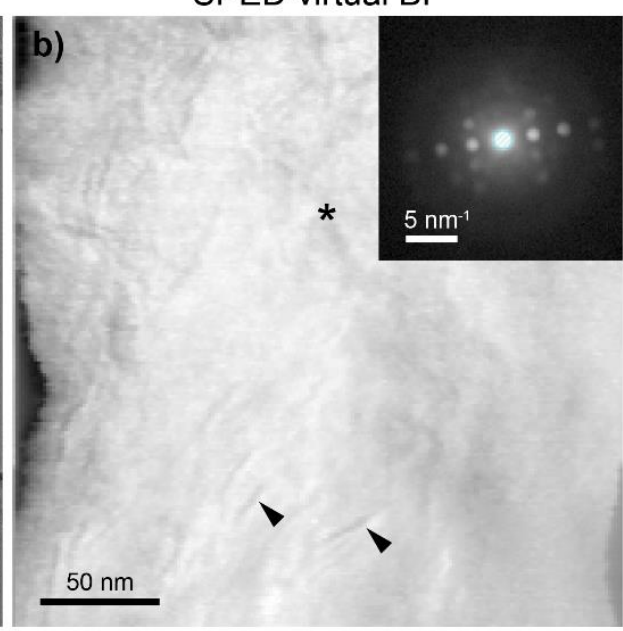

SPED virtual DF

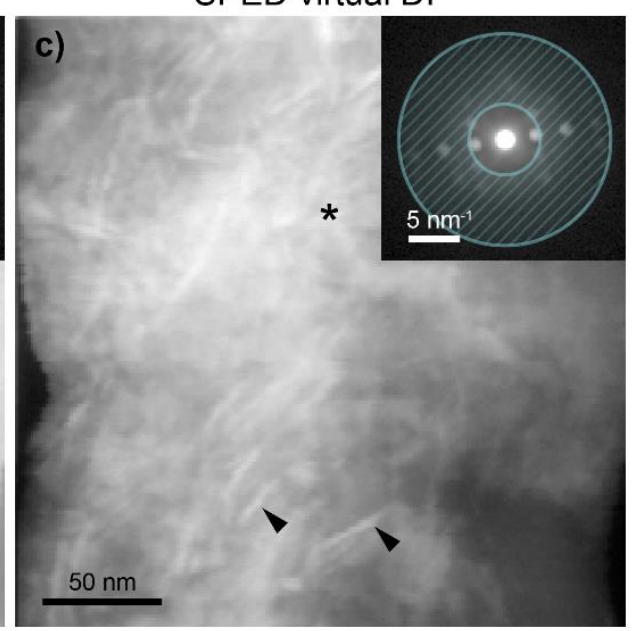

Figure 9: a) TEM bright-field image of the sample analysed in SPED. Virtual b) bright-field and c) dark-field reconstructions from the SPED scan. Insets show exemplary electron diffraction patterns (location indicated by asterisks) and apertures used for the virtual reconstructions (cyan shading). Arrowheads indicate selected crystals in the three respective micrographs.

In a next step of the analysis, crystal phase and orientation were identified. To identify and map crystalline phases, it was necessary to match observed diffraction patterns to the candidate phases. Based on the spectroscopy results (Figure 6), these candidates were OCP and HA in all possible orientations. This matching was done manually by comparing the main characteristics 
of the observed patterns with simulated patterns of the candidate phases (Appendix 5). Analysis of the DPs captured during the SPED scan revealed that the observed particles are crystalline and match well with simulated DPs of HA (Figure 10a-f).

Further analysis of the DPs revealed that the HA crystals were present in various principal orientations. Crystals with a reciprocal $c^{*}$-axis perpendicular to the image plane (zone axis [001]) featured hexagonal diffraction patterns with reflections of the $\{300\}$-type at $d_{300^{-1}}=3.67 \mathrm{~nm}^{-1}$. Crystals with $c^{*}$ parallel to the image plane [uv0] exhibited $(00 l)$ reflections with $l=2 n$, the first one observed at $d_{002^{-1}}=2.91 \mathrm{~nm}^{-1}$. Crystals with the $a^{*}$ or $b^{*}$-axis parallel to the sample plane showed a pattern with a row of $\{300\}$ reflections. Furthermore, SPED data allowed us to analyse crystal orientation in more detail, for example to correlate crystal morphology and lattice orientation. This is especially useful for crystals oriented with $c^{*}$ parallel to the sample plane. The real-space $c$-axis orientations are labelled in the insets in Figure 10i. Surprisingly, the detected $c$-axes did not coincide with the short axis of the crystals. 
$c^{*} \perp$ img, e.g. [001] $\quad c^{*} \angle$ img, e.g. [031] $\quad c^{*} \|$ img, e.g. [170]
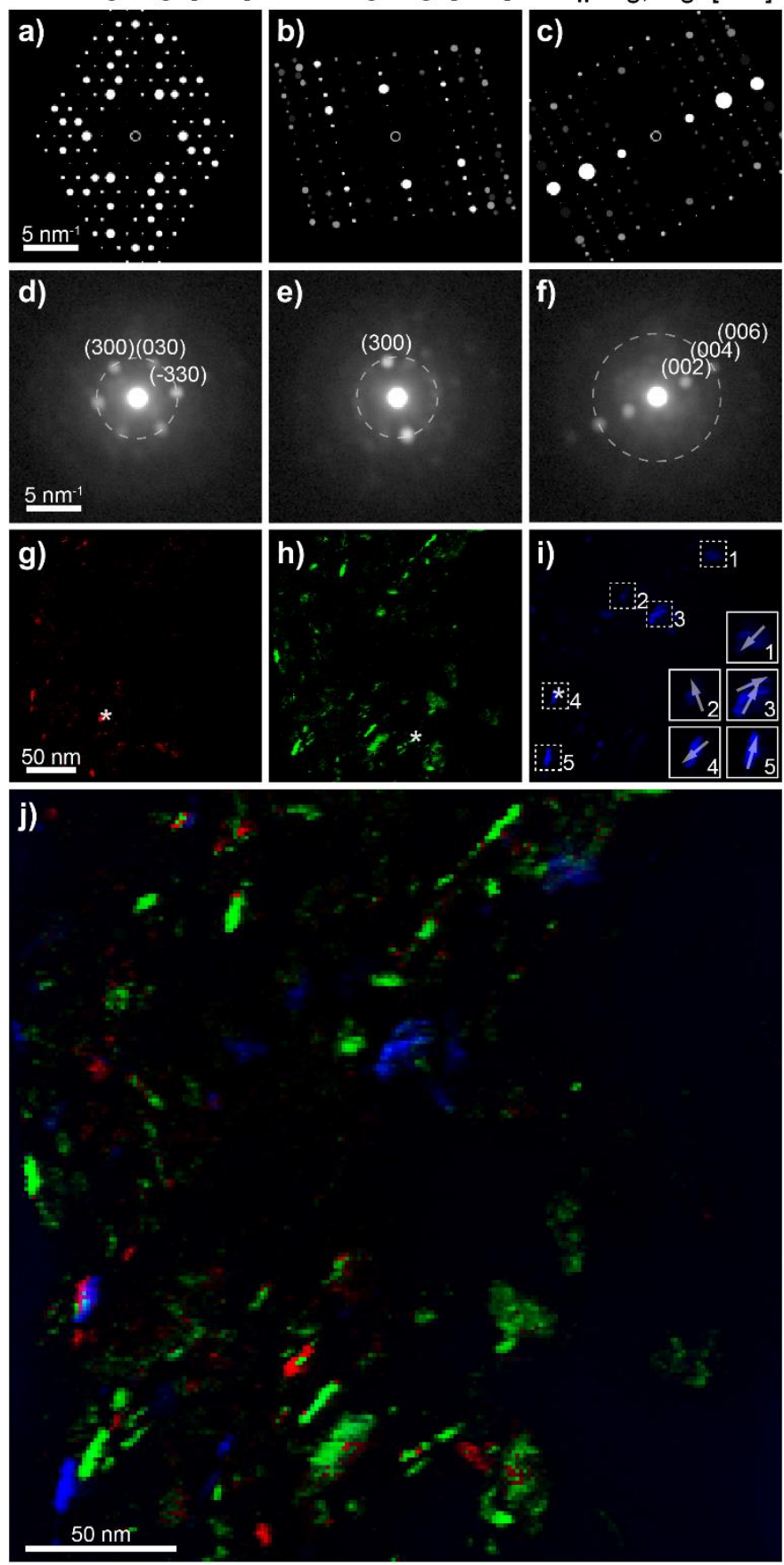

Figure 10: a-c) Simulated DPs for HA matched well with d-f) DPs observed in SPED for various zone axes.

Crystals were mapped with respect to their $c^{*}$-axis orientation, which can either be perpendicular (a, d, g), tilted (b, e, h), or parallel (c, f, i) to the image plane. g-i) Reconstructions for the different principal orientations are shown in as intensity maps. Asterisks indicate locations of the exemplary DPs. Insets (2x magnification) in i) show the $c$-axis orientation of selected nanocrystals that have a $c^{*}$-axis parallel to the image plane. $\mathrm{j}$ ) A mapping overlay of the three principal directions reveals no texture in crystal orientation. 


\section{Discussion}

In the present study, synthetic hydrogel matrices were mineralised with ACP in a one-step process. A set of complementary methods was used to investigate the stabilisation of the formed ACP via two principally different approaches, surface adsorption of citrate and ionic substitution with zinc. The performed experiments help to understand the precipitation and transformation processes in synthetic hydrogel networks in the presence and absence of such stabilisers.

\subsection{Initial mineral precipitation}

The CaP phase initially precipitating from a supersaturated solution of calcium and orthophosphate ions depends on a dynamic interplay of several parameters, most importantly $\mathrm{pH}$, ion concentration, and the presence of stabilisers. At neutral or alkaline $\mathrm{pH}, \mathrm{ACP}$ is typically the first $\mathrm{CaP}$ phase to precipitate from a supersaturated solution of calcium and orthophosphate ions due to its low surface energy [21, 24]. Additionally, the strong interaction between $\mathrm{Ca}^{2+}$ and phosphate anions leads to the rapid formation of thermodynamically metastable ACP particles lacking long-range atomic order [19]. At acidic pH, however, crystalline phases such as OCP or DCPD can precipitate directly $[49,50]$. The presence of stabilisers, high supersaturation, and a high $\mathrm{Ca} / \mathrm{P}$ ratio have all been reported to favour the precipitation of ACP over crystalline phases, even under acidic conditions [50-52]. The fact that, despite the acidic $\mathrm{pH}$, we observed initial precipitation of ACP even in the NoAdd group, suggests that this is because of the high supersaturation and $\mathrm{Ca} / \mathrm{P}$ ratio.

Interestingly, the morphology and size of ACP particles varied slightly depending on the additives. In general, the morphology of the initially precipitated ACP particles matches well with previous findings, which describe it as "featureless, spherical particles" of 20-200 nm diameter [24]. Smaller ACP particles of 60-70 $\mathrm{nm}$ diameter were reported in the presence of 
PEG [25]. Notably, ACP particles observed in Zn10 samples featured a very smooth surface morphology without any signs of crystallisation. In NoAdd and Cit50 samples, on the other hand, ACP particles appeared more particulate, resembling particles at an early stage of ACP to HA transformation reported by Eanes et al. [21].

FTIR spectra of Zn10 samples after 20 minutes in buffer exhibited a characteristic broad ACP peak $[24,25,47,53]$. Spectra obtained from the same samples ten minutes earlier or from Cit50 samples, in contrast, showed a much less pronounced, more structured absorbance band. The reason for this might be the lower $\mathrm{pH}$ of these samples, as the spectra in fact resemble those reported for ACP at $\mathrm{pH} 6.0$ [50].

Five minutes after mixing, composites were flushed with Tris- $\mathrm{HCl}$ buffer to mimic the injection into a biological tissue. Flushing has two direct effects on the composites: an outwards diffusion of unprecipitated ions and reaction buffer, and an inwards diffusion of flushing buffer leading to a gradual increase in $\mathrm{pH}$ beginning at the buffer-composite interface. The system becomes locally supersaturated and additional mineral precipitates close to the interface, both inside and outside the hydrogel matrix. We observed this flushing induced precipitation in DFLM and CLSM (Figure 3). The outwards diffusion of unprecipitated ions was also evident in the release of $\sim 57 \%$ of the total calcium within the first ten minutes in buffer (Figure 4). This is a consequence of the high $\mathrm{Ca} / \mathrm{P}$ ratio of 2.0 , which resulted in an excess of $\mathrm{Ca}$, and the relatively high solubility of $\mathrm{Ca}$ and phosphate at the acidic $\mathrm{pH}$ in the composites before buffering. Remarkably, comparatively little zinc was released from Zn10 composites during flushing, indicating that it is preferentially incorporated in the precipitated mineral. This is likely a consequence of the strong affinity between zinc and phosphate ions [32], which also explains the faster precipitation rate in $\mathrm{Zn} 10$ composites and the related fast initial $\mathrm{pH}$ drop.

The sustained release of calcium or calcium and zinc following the initial burst release suggests that mineral is slowly dissolved. Interestingly, this occurred at almost the same rate for all 
sample groups, despite the differences in mineral phase. In vitro studies are required to exclude potential cytotoxic effects of the released zinc. However, the slow and steady release rate together with the minimal burst release suggest that cytotoxic concentrations can be avoided. Additionally, the reported release rates were observed in calcium- and phosphate-free buffer, whereas blood serum typically contains 2.6 and $0.5 \mathrm{mM}$ calcium and phosphate, respectively [54]. Flushing gels with a medium containing calcium and phosphate ion would result in a lower ion concentration gradient and hence a reduced outwards diffusion of calcium and zinc ions from the composites. More mineral would precipitate inside the matrices as the supersaturation increases with increasing $\mathrm{pH}$. Furthermore, the effect of blood proteins, which was neglected in this proof of concept study, will influence the mineralisation process [23].

\subsection{Crystallisation and transformation in studied composite groups}

Different mineral phases and transformations were found for additive-free, citrate stabilised, and zinc-substituted composites. Figure 11 schematically summarises these transformations. Findings for the different composite groups will be discussed in detail below. 


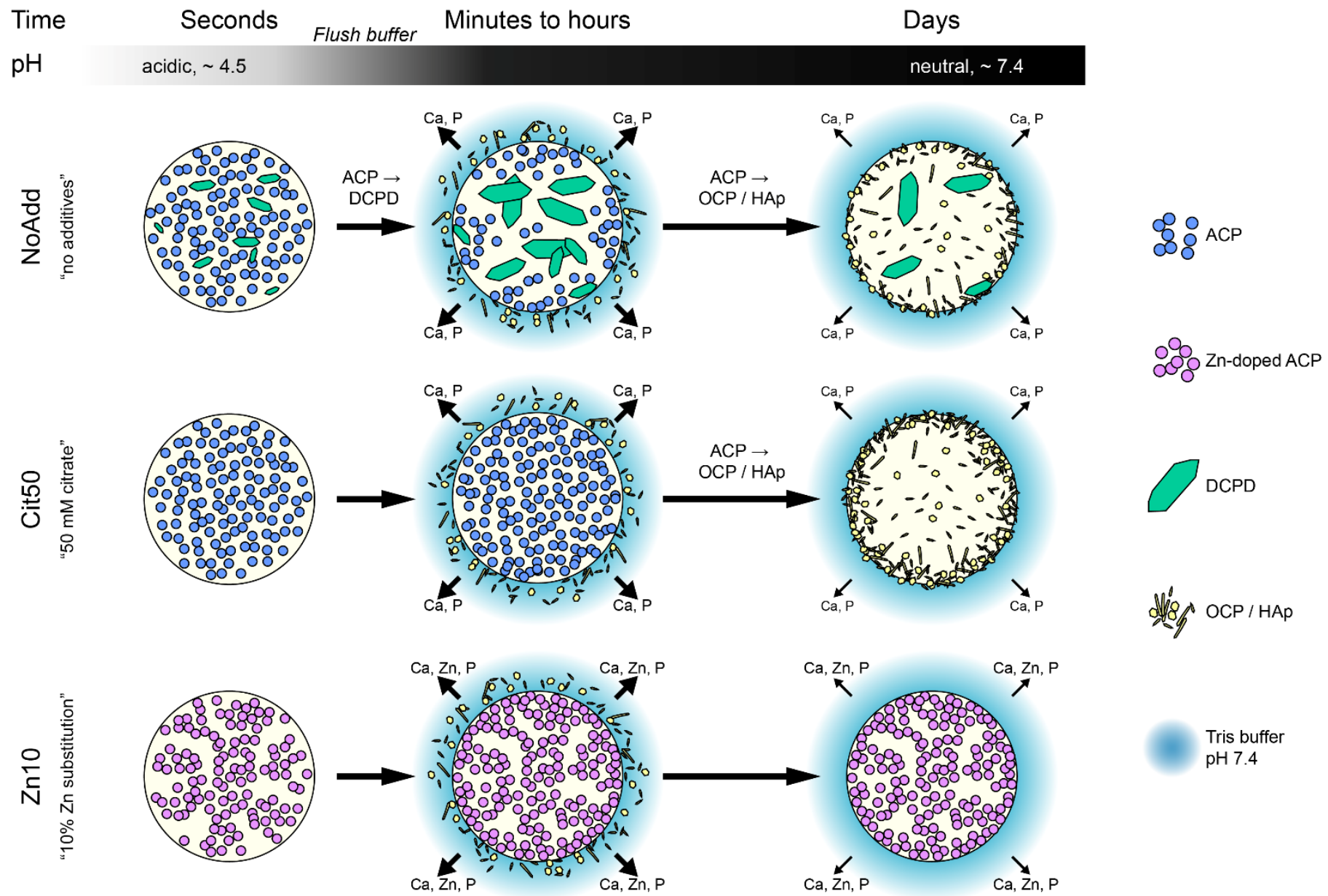

Figure 11: Schematic representation of proposed mineral transformations in presented PEG-calcium phosphate composites.

\subsubsection{NoAdd}

Although previous reports found that $\mathrm{ACP}$ could be stabilised in the presence of large amounts of PEG via formation of a $\mathrm{C}-\mathrm{O}-\mathrm{C} \mathrm{Ca}$ (II) complex [25], we found no such effect preventing the fast formation and growth of brushite crystals under the tested conditions. This might be due to the relatively low PEG concentration of $50 \mu \mathrm{g} / \mathrm{ml}$, the fact that the polymer is bound in a threedimensional network, and the low $\mathrm{pH}$ increasing both the solubility of $\mathrm{ACP}$ and the nucleation rate of brushite [55]. Large brushite crystals formed predominantly at the surface of the hydrogel matrix. We assume that this is due to the physical growth restriction inside the 
network. During ageing in buffer, we found that crystals easily detached from the composite surface, explaining the rapid drop in intensity observed for the DCPD signal in ATR-FTIR.

Due to the acidic $\mathrm{pH}$, it is reasonable to assume that the initially formed ACP features a low $\mathrm{Ca} / \mathrm{P}$ ratio. The low $\mathrm{Ca} / \mathrm{P}$ ratio suggests that the transformation of $\mathrm{ACP}$, which has not been consumed by early brushite crystals, into HA did not proceed in situ, but via a dissolution reprecipitation pathway $[21,53]$. This solution-mediated transformation would be in agreement with the observation that crystalline phases were formed predominantly at the periphery of the composites simultaneously to the dissolution of ACP in the core as observed in SEM and vibrational spectroscopy. Assuming that the initial ACP featured a low $\mathrm{Ca} / \mathrm{P}$ ratio, it is furthermore reasonable to assume that the transformation into HA proceeded via OCP as an intermediate phase [53]. This again is in good agreement with the spectroscopic observations, which indicated OCP or a mixed OCP/HA phase after three days of curing. CRM showed that the $v_{1}\left(\mathrm{PO}_{4}\right)$ peak was shifted towards higher wavenumbers compared to Cit50 samples. Assuming that mineral is transformed in an ACP $\rightarrow$ OCP $\rightarrow$ OCP/HA $\rightarrow$ HA sequence, NoAdd samples show a higher degree of maturation.

\subsubsection{Cit50}

In contrast to additive free composites, citrate effectively prevented brushite formation and stabilised the precipitated ACP for at least several hours. In SEM images, ACP particles appeared less grainy and more spherical compared to additive-free samples (Figure 2d\&e), indicating that citrate successfully adsorbed to ACP, retarding surface nucleation.

After three days in buffer, the densely mineralised region close to the composite edge clearly contained crystalline $\mathrm{CaP}$. At the same time, electron and light micrographs showed that little mineral remained in the centre of the sample. This can possibly be explained by the high density of nucleation sites close to the hydrogel edge, where additional mineral precipitated during flushing. Furthermore, we presume that less free citrate is available to adsorb and stabilise the 
ACP formed during flushing, making these particles the preferred nuclei for crystallisation. The formed crystalline phase appears to be OCP or a mixture of OCP/HA based on spectroscopy $[56,57]$. An exact distinction of these phases based on morphology or spectroscopic data is difficult due to their close structural similarity [58]. However, closer inspection of the peak shape revealed a main peak at $954 \mathrm{~cm}^{-1}$ with a shoulder at $959 \mathrm{~cm}^{-1}$. After extended ageing for 20 days, this converted into a single peak at $959 \mathrm{~cm}^{-1}$ (Figure 6b). We have previously been able to identify a similar pattern for the OCP to HA transformation [22]. This would also be in agreement with the well-established nature of OCP as a kinetically favoured intermediate phase in the ACP to HA transformation [20, 59]. As the OCP to HA transformation is gradual, mixed phases commonly exist [60]. Based on the Raman spectra, it is reasonable to assume that we observe such a mixed phase predominantly composed of OCP after three days of ageing (Figure $6 b)$.

Unexpectedly, though, SPED analysis identified the mineral as HA. A possible explanation for this might be the extensive sample preparation before SPED analysis. In situ transformation of OCP to HA in the absence of water has previously been reported [61] and might occur during the three-day resin curing process at $60^{\circ} \mathrm{C}$. This seems especially plausible, as some DPs that were assigned to [1-10 $]_{\mathrm{HA}}$ feature faint reflections resembling a DP of [110] $\mathrm{OCP}$, which would correspond with the reported alignment for the OCP to HA transformation (Figure A 4). It might further explain the fact that the observed $c$-axes did not coincide with the short axes of the HA crystals as expected, as the crystal morphology might be abnormal due to constricted growth in the resin. Using an embedding resin that cures at low-temperatures might help to reduce such phase transformations during sample preparation. It is unlikely that the mineral phase transformation was induced by beam damage as the short dwell-time of the electron beam during the SPED scan and a small probe-forming aperture, which results in a low electron dose compared to conventional and high-resolution TEM. Altogether, optimised sample preparation 
combined with a better detection, might lead to better contrast in the DPs and automated template matching may become viable [62].

\subsubsection{Zn10}

The zinc-doped ACP was very stable and did not show any signs of crystallisation for up to 20 days. In Tris-HCl buffer, the amorphous particles appeared to steadily dissolve homogeneously throughout the composite. Notably, the $\mathrm{Zn}$ incorporation rates in ACP achieved here are very high compared to findings reported elsewhere [33]. Considering the concentrations of calcium and zinc remaining in the composites after flushing, we found a $\mathrm{Zn} / \mathrm{Ca}$ ratio of $19.6 \%$. This ratio increased in a linear fashion over $21.2 \%$ after one day to $25.7 \%$ after 3 days of ageing. This suggests that calcium is preferentially dissolved from the particles via surface erosion evident in SEM images, which is in accordance with previously reported findings for Zn-doped ACP [63]. Additionally, preliminary experiments showed that the crystallisation inhibition was dependent on the $\mathrm{Zn}$ concentration, as lower $\mathrm{Zn}$ substitution was insufficient to prevent early crystallisation (Figure A 5).

\section{Conclusions}

We demonstrated the formation of hydrogel-CaP composites in a simple one-step procedure. In the absence of additives, initially formed ACP was partly consumed by fast growing brushite crystals, while remaining ACP transformed into poorly crystalline HA. Brushite formation was inhibited in the presence of both citrate and zinc. Citrate-stabilised ACP gradually converted into HA within a time period of approximately 3-20 days. Zinc was successfully incorporated into the amorphous phase, producing very stable zinc-doped ACP that did not show any signs of transformation within a 20 day period. The presented crystallisation inhibition strategies provide a simple toolset to form ACP of tailorable stability in synthetic organic matrices. The fact that composites containing ACP were formed under mildly acidic conditions should enable 
the system to function under inflammatory conditions that are commonly found in bone defects. The potential of the presented composites for the application as bone scaffolds needs to be investigated in further studies.

A comprehensive set of complementary microscopic and spectroscopic techniques allowed us to study mineral formation and transformation processes within the composites in native conditions. Scanning precession electron diffraction provided additional information about the mineral composition and orientation on the nano-scale. This method has not been previously employed to characterise complex samples such as organic-inorganic composites and holds great promise for the advanced assessment of the nanostructure in hydrogel-CaP composites.

\section{Acknowledgements}

The authors would like to thank Ragnhild Sæterli for her assistance with the SPED experiments, Antje Hofgaard at the EM lab for biological sciences at the IBV at UiO for preparing the TEM sample, and Olivia Lasky for proof reading the manuscript. This work was supported by the Osteology Foundation (grant number 15-091); the Research Council of Norway (FRINATEK, grant numbers 214607 and 231530); and travel grants awarded by the Scandinavian Society for Biomaterials and BioStruct. The Research Council of Norway is acknowledged for the support to the Norwegian Micro- and Nano-Fabrication Facility (NorFab, grant number 245963/F50); and NORTEM (grant number 197405).

\section{References}

[1] P.V. Giannoudis, H. Dinopoulos, E. Tsiridis, Bone substitutes: an update, Injury 36 Suppl 3(3, Supplement) (2005) 20-27.

[2] T.T. Roberts, A.J. Rosenbaum, Bone grafts, bone substitutes and orthobiologics: the bridge between basic science and clinical advancements in fracture healing, Organogenesis 8(4) (2012) 114-124.

[3] E.D. Arrington, W.J. Smith, H.G. Chambers, A.L. Bucknell, N.A. Davino, Complications of iliac crest bone graft harvesting, Clin. Orthop. Relat. Res. 329 (1996) 300-309. 
[4] W.W. Tomford, Transmission of Disease through Transplantation of Musculoskeletal Allografts, J. Bone Jt. Surg., Am. Vol. 77a(11) (1995) 1742-1754.

[5] K. Gkioni, S.C. Leeuwenburgh, T.E. Douglas, A.G. Mikos, J.A. Jansen, Mineralization of hydrogels for bone regeneration, Tissue Eng Part B Rev 16(6) (2010) 577-585.

[6] M.P. Lutolf, F.E. Weber, H.G. Schmoekel, J.C. Schense, T. Kohler, R. Muller, J.A. Hubbell, Repair of bone defects using synthetic mimetics of collagenous extracellular matrices, Nat. Biotechnol. 21(5) (2003) 513-518.

[7] S. Fu, P. Ni, B. Wang, B. Chu, L. Zheng, F. Luo, J. Luo, Z. Qian, Injectable and thermosensitive PEG-PCL-PEG copolymer/collagen/n-HA hydrogel composite for guided bone regeneration, Biomaterials 33(19) (2012) 4801-4809.

[8] M. Patel, M.W. Betz, E. Geibel, K.J. Patel, J.F. Caccamese, D.P. Coletti, J.J. Sauk, J.P. Fisher, Cyclic Acetal Hydroxyapatite Nanocomposites for Orbital Bone Regeneration, Tissue Eng., Part A 16(1) (2009) 55-65.

[9] A.S. Posner, F. Betts, Synthetic Amorphous Calcium-Phosphate and Its Relation to BoneMineral Structure, Acc. Chem. Res. 8(8) (1975) 273-281.

[10] N.C. Blumenthal, A.S. Posner, J.M. Holmes, Effect of preparation conditions on the properties and transformation of amorphous calcium phosphate, Mater. Res. Bull. 7(11) (1972) 1181-1189.

[11] M. Murshed, Mechanism of Bone Mineralization, Cold Spring Harbor Perspect. Med. Epub ahead of print (2018) 1-11.

[12] M. Bottini, S. Mebarek, K.L. Anderson, A. Strzelecka-Kiliszek, L. Bozycki, A.M.S. Simao, M. Bolean, P. Ciancaglini, J.B. Pikula, S. Pikula, D. Magne, N. Volkmann, D. Hanein, J.L. Millan, R. Buchet, Matrix vesicles from chondrocytes and osteoblasts: Their biogenesis, properties, functions and biomimetic models, Biochim. Biophys. Acta, Gen. Subj. 1862(3) (2018) 532-546.

[13] J. Mahamid, A. Sharir, D. Gur, E. Zelzer, L. Addadi, S. Weiner, Bone mineralization proceeds through intracellular calcium phosphate loaded vesicles: a cryo-electron microscopy study, J. Struct. Biol. 174(3) (2011) 527-535.

[14] S. Boonrungsiman, E. Gentleman, R. Carzaniga, N.D. Evans, D.W. McComb, A.E. Porter, M.M. Stevens, The role of intracellular calcium phosphate in osteoblast-mediated bone apatite formation, Proc. Natl. Acad. Sci. USA 109(35) (2012) 14170-14175.

[15] C. Shao, R. Zhao, S. Jiang, S. Yao, Z. Wu, B. Jin, Y. Yang, H. Pan, R. Tang, Citrate Improves Collagen Mineralization via Interface Wetting: A Physicochemical Understanding of Biomineralization Control, Adv. Mater. 30(8) (2018) 1704876.

[16] J. Mahamid, A. Sharir, L. Addadi, S. Weiner, Amorphous calcium phosphate is a major component of the forming fin bones of zebrafish: Indications for an amorphous precursor phase, Proc. Natl. Acad. Sci. USA 105(35) (2008) 12748-12753.

[17] S.V. Dorozhkin, Amorphous calcium (ortho)phosphates, Acta Biomater. 6(12) (2010) 4457-4475.

[18] L.L. Hench, J.M. Polak, Third-generation biomedical materials, Science 295(5557) (2002) 1014-1017.

[19] E.D. Eanes, I.H. Gillessen, A.S. Posner, Intermediate States in the Precipitation of Hydroxyapatite, Nature 208 (1965) 365-367. 
[20] J.C. Elliott, General Chemistry of the Calcium Orthophosphates, in: J.C. Elliott (Ed.), Structure and Chemistry of the Apatites and Other Calcium Orthophosphates, Elsevier, Amsterdam, 1994, pp. 1-62.

[21] E.D. Eanes, J.D. Termine, M.U. Nylen, An electron microscopic study of the formation of amorphous calcium phosphate and its transformation to crystalline apatite, Calcif. Tissue Res. 12(2) (1973) 143-158.

[22] S.H. Bjornoy, D.C. Bassett, S. Ucar, B.L. Strand, J.P. Andreassen, P. Sikorski, A correlative spatiotemporal microscale study of calcium phosphate formation and transformation within an alginate hydrogel matrix, Acta Biomater. 44 (2016) 254-266.

[23] S. Mann, Chemical control of biomineralization, Biomineralization: principles and concepts in bioinorganic materials chemistry, Oxford University Press, Oxford, 2001, pp. 3867.

[24] S.V. Dorozhkin, Calcium Orthophosphates in Nature, Biology and Medicine, Materials 2(2) (2009) 399-498.

[25] Y. Li, W. Weng, In vitro synthesis and characterization of amorphous calcium phosphates with various Ca/P atomic ratios, J. Mater. Sci. Mater. Med. 18(12) (2007) 2303-2308.

[26] E. Boanini, M. Gazzano, A. Bigi, Ionic substitutions in calcium phosphates synthesized at low temperature, Acta Biomater. 6(6) (2010) 1882-1894.

[27] F. Dickens, The citric acid content of animal tissues, with reference to its occurrence in bone and tumour, Biochem. J. 35(8-9) (1941) 1011-1023.

[28] E. Davies, K.H. Muller, W.C. Wong, C.J. Pickard, D.G. Reid, J.N. Skepper, M.J. Duer, Citrate bridges between mineral platelets in bone, Proc. Natl. Acad. Sci. USA 111(14) (2014) E1354-E1363.

[29] Y. Chen, W.J. Gu, H.H. Pan, S.Q. Jiang, R.K. Tang, Stabilizing amorphous calcium phosphate phase by citrate adsorption, CrystEngComm 16(10) (2014) 1864-1867.

[30] J.M. Aitken, Factors affecting the distribution of zinc in the human skeleton, Calcif. Tissue Res. 20(1) (1976) 23-30.

[31] M. Yamaguchi, Role of nutritional zinc in the prevention of osteoporosis, Mol. Cell. Biochem. 338(1-2) (2010) 241-254.

[32] A. Bigi, E. Foresti, M. Gandolfi, M. Gazzano, N. Roveri, Inhibiting Effect of Zinc on Hydroxylapatite Crystallization, J. Inorg. Biochem. 58(1) (1995) 49-58.

[33] K.A. Gross, L. Komarovska, A. Viksna, Efficient zinc incorporation into hydroxyapatite through crystallization of an amorphous phase could extend the properties of zinc apatites, J. Aust. Ceram. Soc. 49(2) (2013) 129-135.

[34] N. Kanzaki, K. Onuma, G. Treboux, S. Tsutsumi, A. Ito, Inhibitory effect of magnesium and zinc on crystallization kinetics of hydroxyapatite (0001) face, J. Phys. Chem. B 104(17) (2000) 4189-4194.

[35] T.A. Fuierer, M. LoRe, S.A. Puckett, G.H. Nancollas, A Mineralization Adsorption and Mobility Study of Hydroxyapatite Surfaces in the Presence of Zinc and Magnesium Ions, Langmuir 10(12) (1994) 4721-4725.

[36] A. Ito, K. Ojima, H. Naito, N. Ichinose, T. Tateishi, Preparation, solubility, and cytocompatibility of zinc-releasing calcium phosphate ceramics, J. Biomed. Mater. Res. 50(2) (2000) 178-183. 
[37] I.Y. Ortiz, A.R. dos Santos, A.M. Costa, E. Mavropoulos, M.N. Tanaka, M.H.P. da Silva, S.D. Camargo, In vitro assessment of zinc apatite coatings on titanium surfaces, Ceram. Int. 42(14) (2016) 15502-15510.

[38] H. Kawamura, A. Ito, T. Muramatsu, S. Miyakawa, N. Ochiai, T. Tateishi, Long-term implantation of zinc-releasing calcium phosphate ceramics in rabbit femora, J. Biomed. Mater. Res. A 65(4) (2003) 468-474.

[39] H.J. Seo, Y.E. Cho, T. Kim, H.I. Shin, I.S. Kwun, Zinc may increase bone formation through stimulating cell proliferation, alkaline phosphatase activity and collagen synthesis in osteoblastic MC3T3-E1 cells, Nutr. Res. Pract. 4(5) (2010) 356-361.

[40] J. Patterson, J.A. Hubbell, Enhanced proteolytic degradation of molecularly engineered PEG hydrogels in response to MMP-1 and MMP-2, Biomaterials 31(30) (2010) 7836-7845.

[41] S.H. Bjornoy, S. Mandaric, D.C. Bassett, A.K. Slund, S. Ucar, J.P. Andreassen, B.L. Strand, P. Sikorski, Gelling kinetics and in situ mineralization of alginate hydrogels: A correlative spatiotemporal characterization toolbox, Acta Biomater. 44 (2016) 243-253.

[42] M. Schweikle, T. Zinn, R. Lund, H. Tiainen, Injectable synthetic hydrogel for bone regeneration: Physicochemical characterisation of a high and a low $\mathrm{pH}$ gelling system, Mater. Sci. Eng., C 90 (2018) 67-76.

[43] Z. Li, S. Wu, J. Han, S. Han, Imaging of intracellular acidic compartments with a sensitive rhodamine based fluorogenic pH sensor, Analyst 136(18) (2011) 3698-3706.

[44] J. Schindelin, I. Arganda-Carreras, E. Frise, V. Kaynig, M. Longair, T. Pietzsch, S. Preibisch, C. Rueden, S. Saalfeld, B. Schmid, J.Y. Tinevez, D.J. White, V. Hartenstein, K. Eliceiri, P. Tomancak, A. Cardona, Fiji: an open-source platform for biological-image analysis, Nat. Methods 9(7) (2012) 676-682.

[45] F.d.l. Peña, T. Ostasevicius, V.T. Fauske, P. Burdet, E. Prestat, P. Jokubauskas, M. Nord, M. Sarahan, K.E. MacArthur, D.N. Johnstone, J. Taillon, J. Caron, V. Migunov, T. Furnival, A. Eljarrat, S. Mazzucco, T. Aarholt, M. Walls, T. Slater, F. Winkler, B. Martineau, G. Donval, R. McLeod, E.R. Hoglund, I. Alxneit, I. Hjorth, T. Henninen, L.F. Zagonel, A. Garmannslund, 5ht2, hyperspy/hyperspy: HyperSpy 1.3.1, Zenodo, 2018.

[46] M. Klinger, More features, more tools, more CrysTBox, J. Appl. Crystallogr. 50(4) (2017) 1226-1234.

[47] C. Drouet, Apatite formation: why it may not work as planned, and how to conclusively identify apatite compounds, Biomed. Res. Int. 2013 (2013) 490906.

[48] P.A. Midgley, A.S. Eggeman, Precession electron diffraction - a topical review, IUCrJ 2(Pt 1) (2015) 126-136.

[49] F. Abbona, F. Christensson, M.F. Angela, H.E.L. Madsen, Crystal habit and growth conditions of brushite, CaHPO4 · 2H2O, J. Cryst. Growth 131(3-4) (1993) 331-346.

[50] C. Holt, M.J.J.M. Vankemenade, L.S. Nelson, D.W.L. Hukins, R.T. Bailey, J.E. Harries, S.S. Hasnain, P.L. Debruyn, Amorphous Calcium Phosphates Prepared at Ph 6.5 and 6.0, Mater. Res. Bull. 24(1) (1989) 55-62.

[51] H. Furedi-Milhofer, L. Brecevic, B. Purgaric, Crystal growth and phase transformation in the precipitation of calcium phosphates, Faraday Discuss. Chem. Soc. 61(61) (1976) 184-190.

[52] M.J.J.M. van Kemenade, P.L. de Bruyn, A kinetic study of precipitation from supersaturated calcium phosphate solutions, J. Colloid Interface Sci. 118(2) (1987) 564-585. 
[53] C. Combes, C. Rey, Amorphous calcium phosphates: synthesis, properties and uses in biomaterials, Acta Biomater. 6(9) (2010) 3362-3378.

[54] W. Jubiz, J.M. Canterbury, E. Reiss, F.H. Tyler, Circadian rhythm in serum parathyroid hormone concentration in human subjects: correlation with serum calcium, phosphate, albumin, and growth hormone levels, J. Clin. Invest. 51(8) (1972) 2040-2046.

[55] A.L. Boskey, A.S. Posner, Conversion of amorphous calcium phosphate to microcrystalline hydroxyapatite. A pH-dependent, solution-mediated, solid-solid conversion, J. Phys. Chem. 77(19) (1973) 2313-2317.

[56] H. Tsuda, J. Arends, Raman spectroscopy in dental research: a short review of recent studies, Adv. Dental Res. 11(4) (1997) 539-547.

[57] N.J. Crane, V. Popescu, M.D. Morris, P. Steenhuis, M.A. Ignelzi, Raman spectroscopic evidence for octacalcium phosphate and other transient mineral species deposited during intramembranous mineralization, Bone 39(3) (2006) 434-442.

[58] S. Mann, Biomineral types and functions, Biomineralization: principles and concepts in bioinorganic materials chemistry, Oxford University Press, Oxford, 2001, pp. 6-23.

[59] M.B. Tomson, G.H. Nancollas, Mineralization kinetics: a constant composition approach, Science 200(4345) (1978) 1059-1060.

[60] J.C. Elliott, Hydroxyapatite and Nonstoichiometric Apatites, in: J.C. Elliott (Ed.), Structure and Chemistry of the Apatites and Other Calcium Orthophosphates, Elsevier, Amsterdam, 1994, pp. 111-189.

[61] R.L. Xin, Y. Leng, N. Wang, In situ TEM examinations of octacalcium phosphate to hydroxyapatite transformation, J. Cryst. Growth 289(1) (2006) 339-344.

[62] E.F. Rauch, L. Dupuy, Rapid spot diffraction patterns idendification through template matching, Arch. Metall. Mater. 50(1) (2005) 87-99.

[63] P.T. Olesen, T. Steenberg, E. Christensen, N.J. Bjerrum, Electrolytic deposition of amorphous and crystalline zinc-calcium phosphates, J. Mater. Sci. 33(12) (1998) 3059-3063. 